\title{
Danirixin: A Reversible and Selective Antagonist of the CXC Chemokine Receptor 2
}

\author{
Jakob Busch-Petersen, ${ }^{1}$ Donald C. Carpenter, Miriam Burman, ${ }^{2}$ James Foley, \\ Gerald E. Hunsberger, David J. Kilian, Michael Salmon, ${ }^{3}$ Ruth J. Mayer, ${ }^{4}$ John G. Yonchuk, \\ and Ruth Tal-Singer
}

GSK R\&D, King of Prussia PA

Received February 15, 2017; accepted June 9, 2017

\begin{abstract}
CXC chemokine receptor 2 (CXCR2) is a key receptor in the chemotaxis of neutrophils to sites of inflammation. The studies reported here describe the pharmacological characterization of danirixin, a CXCR2 antagonist in the diaryl urea chemical class. Danirixin has high affinity for CXCR2, with a negative log of the $50 \%$ inhibitory concentration $\left(\mathrm{plC}_{50}\right)$ of 7.9 for binding to Chinese hamster ovary cell (CHO)-expressed human CXCR2, and 78-fold selectivity over binding to $\mathrm{CHO}$-expressed CXCR1. Danirixin is a competitive antagonist against CXCL8 in $\mathrm{Ca}^{2+}$-mobilization assays, with a $\mathrm{K}_{\mathrm{B}}$ (the concentration of antagonist that binds $50 \%$ of the receptor population) of $6.5 \mathrm{nM}$ and antagonist potency $\left(\mathrm{pA}_{2}\right)$ of 8.44 , and is fully reversible in washout experiments over 180 minutes. In rat and human whole-blood studies assessing neutrophil
\end{abstract}

activation by surface CD11b expression following CXCL2 (rat) or CXCL1 (human) challenge, danirixin blocks the CD11b upregulation with $\mathrm{plC}_{50}$ s of 6.05 and 6.3, respectively. Danirixin dosed orally also blocked the influx of neutrophils into the lung in vivo in rats following aerosol lipopolysaccharide or ozone challenge, with median effective doses $\left(E D_{50} \mathrm{~s}\right)$ of 1.4 and $16 \mathrm{mg} / \mathrm{kg}$ respectively. Thus, danirixin would be expected to block chemotaxis in disease states in which neutrophils are increased in response to inflammation, such as pulmonary diseases. In comparison with navarixin, a CXCR2 antagonist from a different chemical class, the binding characterization of danirixin is distinct. These observations may offer insight into the previously observed clinical differences in induction of neutropenia between these compounds.

\section{Introduction}

Sustained neutrophil influx to the lung plays a key role of the pathology of diseases such chronic obstructive pulmonary disease (COPD), severe asthma, cystic fibrosis, acute lung injury, and respiratory syncytial virus infection (Wareing et al., 2007; Chapman et al., 2009; Stadtmann and Zarbock, 2012). In these conditions, tissue cells at the site of injury produce ELR+ chemokines (containing a Glu-Leu-Arg motif before the first cysteine), which in turn activate neutrophils through interaction with the G protein-coupled CXC chemokine receptor 1 (CXCR1) and CXC chemokine receptor 2 (CXCR2). The ELR+ chemokines are CXCL1 through CXCL3 and CXCL5 through CXCL8. Chronic exposure to activated neutrophils leads to tissue damage either directly through degranulation of proteases or indirectly through additional cell recruitment, and CXCR2 and its ligands

This work was funded by GSK R\&D. Primary laboratory of origin: GSK R\&D, Respiratory Therapy Area Unit.

${ }^{1}$ Current affiliation: External innovation, Roche, Basel, Switzerland

${ }^{2}$ Current affiliation: GSK Retiree

${ }^{3}$ Current affiliation: Immunology Department, Merck Research Laboratories, Boston, MA, USA

${ }^{4}$ Current affiliation: GSK Retiree

https://doi.org/10.1124/jpet.117.240705. have been implicated in disease processes (Qiu et al., 2003; Quint and Wedzicha, 2007; Wareing et al., 2007; Nagarkar et al., 2009).

CXCR2 and CXCR1 have overlapping and distinct functions (Hartl et al., 2007; Raghuwanshi et al., 2012). All ELR+ chemokines activate CXCR2, whereas only a subset, namely CXCL6, CXCL8, and potentially CXCL7, activate the CXCR1 receptor. Both CXCR1 and CXCR2 recruit neutrophils to sites of inflammation; CXCR2 may be primarily responsible for the initial recruitment of neutrophils, and CXCR1 appears to be responsible for neutrophil movement and some functions, such as respiratory burst and phospholipase D activation. CXCR1 and CXCR2 are also expressed on a number of other cells involved in inflammation, including monocytes, macrophages, lymphocytes, mast cells, dendritic cells, and endothelial cells. Blockade of CXCR2 alone is therefore hypothesized to be key for the prevention of neutrophil migration to sites of inflammation. CXCR2 antagonists studied to date have varying selectivity with respect to CXCR1, and all block neutrophil migration. Additional consequences of more or less selective compounds, such as host defense functions, have not been studied in detail in the clinic at this time.

Challenges in studying ELR+ chemokine-mediated neutrophil function in rodents are ligand and receptor functions of

ABBREVIATIONS: BAL, bronchoalveolar lavage; $\mathrm{CHO}$, Chinese hamster ovary cell; $\mathrm{Cl}$, confidence interval; COPD, chronic obstructive pulmonary disease; CXCR1, CXC chemokine receptors 1; CXCR2, CXC chemokine receptor 2; danirixin, 3-\{4-chloro-2-hydroxy-3-[(3S)-piperidine-3sulfonyl]phenyl\}-1-(3-fluoro-2-methylphenyl)urea; DMSO, dimethyl sulfoxide; $\mathrm{ED}_{50}$, median effective dose; FITC, fluorescein isothiocyanate; hSA, human serum albumin; LPS, lipopolysaccharide; navarixin, 2-hydroxy- $N, N$-dimethyl-3-[[2-[[(1R)-1-(5-methylfuran-2-yl)propyl]amino]-3,4-dioxocyclobuten1-yl]amino]benzamide hydrate; RBC, red blood cell; PE, Phycoerythrin. 
CXCR1 homologs that are distinct from human. In rats, a homologous CXCR1 receptor gene has been identified; however, it does not appear to be expressed. In mice, a functional CXCR1 ortholog may exist (Fan et al., 2007); however, its function and expression have not been sufficiently characterized to draw firm conclusions, and ligand pairing is therefore unknown. In addition, the rat and human ELR + chemokines are distinct; for example, mice and rats lack a CXCL8 ortholog and it is thought that neutrophil chemotaxis in rodents is mediated by CXCL1 [or cytokine-induced neutrophil chemoattractant 1 (CINC-1) in rat] and 2 (or macrophage inflammatory protein 2 in rat) (Fan et al., 2007). CINC-1 levels are upregulated by inflammatory stimuli such as lipopolysaccharide (LPS), ozone, or cigarette smoke. (Haddad et al., 1996; Haddad et al., 2002; Thatcher et al., 2005). In contrast, human LPS challenge upregulates CXCL8 but not CXCL1 (Aul et al., 2012). Thus, in rodent models of inflammation, the functions of ELR + chemokine signaling through these receptors may be significantly different from that observed in humans.

An additional function of CXCR2 has been illuminated from recent human genetic data showing that low frequency missense mutations in CXCR2 are associated with reduced blood leukocyte count. In addition, individuals with frameshift mutations in the CXCR2 gene suffer from myelokathexis or retention of neutrophils in the bone marrow (Auer et al., 2014). $\mathrm{CXCR2}^{-/-}$mice also exhibit this phenotype (Eash et al., 2010). This evidence underscores the importance of CXCR2 in the process of neutrophil migration from the bone marrow.

Numerous small-molecule antagonists of CXCR2 in several chemical series have been reported in the literature (Dwyer and $\mathrm{Yu}, 2014$; compound numbers below are from this reference). Of these, four have been tested in patients with respiratory diseases (cystic fibrosis, asthma, COPD, and bronchiectasis): elubrixin (compound 4; SB-656933), navarixin (compound 11; MK-7123, SCH-527123, PS291811), AZD5069 (compound 27), and danirixin (compound 5; GSK1325756). Danirixin is currently in clinical development for COPD and acute viral respiratory infections. Here we report a detailed pharmacological characterization of danirixin. This compound belongs to the diaryl urea class of CXCR2 antagonists, which also includes previous clinical candidates elubrixin and SB-332235 (Busch-Petersen, 2006). The studies presented here describe the CXCR2 and CXCR1 receptor pharmacology of the compound as well as its effects on neutrophils in vitro and pharmacology in in vivo models of inflammation with special emphasis on preclinical-to-clinical translation.

\section{Materials and Methods}

\section{Compounds}

Danirixin [3-\{4-chloro-2-hydroxy-3-[(3S)-piperidine-3-sulfonyl]phenyl\}-1-(3-fluoro-2-methylphenyl)urea] was synthesized as either freebase (identified as GSK1325756B) or hydrochloride salt (identified as GSK1325756A) by the GSK Respiratory Therapy Area Unit medicinal chemistry group (King of Prussia, PA), as described in Busch-Petersen (2007). Navirixin was prepared following previously reported methods (Dwyer et al., 2006)

\section{Cell Culture}

Each of the receptors CXCR1 and CXCR2 was stably expressed with $\mathrm{G} \alpha 16$ in Chinese hamster ovary cells (CHO)-K1. CHO-K1 cells were grown to 80\% confluence in Dulbecco's modified Eagle's medium/F12
(Ham's) media, 1:1, with $10 \%$ fetal calf serum, $2 \mathrm{mM}$ L-glutamine and $0.2 \mathrm{mg} / \mathrm{ml} \mathrm{G} 418$, at $37^{\circ} \mathrm{C}$ in a $5 \% \mathrm{CO}_{2}$ incubator.

\section{Scintillation Proximity Binding Assays (CXCR2 and CXCR1)}

Membranes were prepared from frozen CHO-K1 cell pellets using a hypotonic buffer containing protease inhibitors as previously described (Sarau et al., 1997; Podolin et al., 2002). Assays were conducted using scintillation proximity with wheat germ agglutinin beads (GE Healthcare Life Sciences, Marlborough, MA). Briefly, membranes were incubated with beads in binding buffer at $4^{\circ} \mathrm{C}$ for 30 minutes. Compounds were diluted 1:20 from 100\% dimethyl sulfoxide (DMSO) solutions in a final volume of $100 \mu$ l containing binding buffer, beads, and $0.225 \mathrm{nM}$ $\left.{ }^{[125} \mathrm{I}\right]$ CXCL8 in Packard OptiPlates (Packard Bioscience Company, Meriden, CT). After a 45-minute incubation, the plates were centrifuged at 2000 rpm for 5 minutes and counted in a Packard TopCount. Specific binding was determined by subtracting the counts observed at $30 \mu \mathrm{M}$ of CXCR2 or CXCR1 antagonist (nonspecific binding) from the total binding counts of CXCR2 or CXCR1, respectively. Curves were generated using GraphPad Prism (v4.0 or later; GraphPad, LaJolla, $\mathrm{CA}$ ) and $\mathrm{IC}_{50}$ was determined as the concentration required to displace $50 \%$ of the specific binding.

\section{$\mathrm{Ca}^{2+}$-Mobilization Assays}

CHO-K1 cells expressing each receptor were cultured and $\mathrm{Ca}^{2+}$ mobilization assays performed in a standard protocol as previously described (Podolin et al., 2002). Compound potency as $\mathrm{pA}_{2}$ was calculated as the ratio of equiactive CXCL8 responses in the presence and absence of a single concentration of antagonist using the formula $\log (\mathrm{DR}-1)-\log [\mathrm{B}]$, where $\mathrm{DR}$ is the dose ratio of $\mathrm{EC}_{50}$ of CXCL8 in the presence and absence of compound at concentration B. Further characterization of the pattern of antagonism was done using classic Schild analysis from a series of curves at a single compound concentration and a range of CXCL8 concentrations. Unconstrained fits included unity within the $95 \%$ confidence limit (CL) for the slope, so values reported are from a fit where slope was constrained to 1. $\mathrm{pA}_{2}$ is the antagonist concentration which causes a two fold shift in concentration-response curve for an agonist (representing antagonist potency); it is the negative logarithm of the $\mathrm{K}^{\mathrm{B}}$ which is the concentration of the antagonist that binds $50 \%$ of the receptor population. Compound reversibility was evaluated in washout studies. For the preincubation steps, cells were incubated with compound containing media and washout cells, or vehicle containing media and nonwashout cells, and incubated for 60 minutes at $37^{\circ} \mathrm{C}$; medium was aspirated and compound or vehicle re-added for 20 minutes, and then for a further 10 minutes after an additional wash (compound treatment of 90 minutes total). For the washout steps, cells were washed with Krebs-Ringer-HEPES buffer by aspiration, followed by a 30-minute incubation in fresh media a total of three times (90 minutes total). Cells were then loaded with $4 \mu \mathrm{M}$ Fluo-4 AM and incubated for 60 minutes. Fluo- 4 media was subsequently aspirated, cells were retreated as appropriate, and incubated for 30 minutes, for a total washout period of 180 minutes, and the standard challenge protocol was followed.

Effect on Agonist-Induced Neutrophil CD11b Expression in Whole Blood. Rat in vitro assay: Fresh rat blood obtained by cardiac puncture into an EDTA tube was incubated with danirixin $(10 \mu \lambda$ added to final concentration $0.03-10 \mu \mathrm{M}$ and $0.1 \%$ DMSO) and rat CXCL2 (10 nM final; PeproTech, Rocky Hill, NJ) at $37^{\circ} \mathrm{C}$ for 30 minutes. The samples were iced for 10 minutes and then labeled with a fluorescein isothiocyanate (FITC)-tagged CD11b-specific antibody (Antigenix America Inc., Huntington Station, NY). The red blood cells (RBCs) were lysed and the leukocytes were washed and fixed. For flow cytometric analysis, LDS-751 was added to label nucleated leukocytes and further exclude any unlysed RBCs. Briefly, threshold in the LDS-751 detector was increased to eliminate RBCs and then an analysis gate was placed around the granulocyte population in the forward scatter/side scatter plot. The FITC mean fluorescence intensity (MFI) of this population was then assessed as CD11b 
expression. A similar assay has been described previously for human blood (Davis et al., 2000).

\section{Rat Ex Vivo Assay}

Following oral dosing of GSK1325756B to male Lewis rats $(n=$ 5 rats per group, see in vivo studies for details), whole blood was obtained by cardiac puncture 1 hour postdosing. Rat pharmacokinetic studies demonstrated a $\mathrm{T}_{\max }$ of 0.25 hours. Therefore, for efficacy and logistical reasons, 1 hour postdose was selected as the optimal time point. Whole blood collected in EDTA tubes was treated with CXCL2 for 20 minutes at $37^{\circ} \mathrm{C}$ and then the cells were labeled with a FITCtagged CD11b-specific antibody (Antigenix America). Analysis by flow cytometry was performed as described above.

\section{Human In Vitro Assay}

Whole blood was obtained from consenting donors into EDTA tubes and incubated with danirixin for 10 minutes prior to treatment with human CXCR1 (10 nM final; PeproTech) for a further 10 minutes, all at $37^{\circ} \mathrm{C}$. Following a light fixation on ice, cells were labeled with a FITC-tagged CD11b-specific antibody (Beckman Coulter, Inc., Brea, CA ) and a PE-tagged CD16 antibody (Dako/Agilent Technologies, Santa Clara, CA). For flow cytometric analysis, LDS-751 was added to label nucleated leukocytes and eliminate RBCs from analysis. Briefly, threshold in the LDS-751 detector was increased to exclude RBCs and then an analysis gate was placed around the granulocyte population in the forward scatter/side scatter plot. A second analysis gate was then placed around the CD16+ neutrophils within the granulocyte gate. The FITC MFI of this population was then assessed as CD11b expression.

\section{Data Analysis}

Percent inhibition was calculated by subtracting each value of the percentage of positive control (value with CXCL2 alone) from 100. For ex vivo studies, the maximal response was defined as the sample value from animals treated with vehicle alone and stimulated with $100 \mathrm{nM}$ CXCL2. $\mathrm{ED}_{50}$ was defined as concentration of CXCL2 required to induce $50 \%$ of the maximal response and was calculated using GraphPad Prism v4.0 from a sigmoidal, four-parameter, nonlinear regression curve with variable slope and constrained between 0 and 100. Samples were excluded from the analysis if there was excessive clumping of cells, activation of cells resulting in high CD11b expression prior to CXCL2 activation, or lack of response to CXCL2 over the dose response. $N$ ranged from 2 to 5 samples per point, typically 4 to 5 .

\section{In Vivo Studies in the Rat}

All studies were conducted in accordance with the GlaxoSmithKline Policy on the Care, Welfare and Treatment of Laboratory Animals and were reviewed by the Institutional Animal Care and Use Committee at GlaxoSmithKline facilities, where the work was performed. Male Lewis rats (275-400 g; Charles River Laboratories, Portage, MI) were used in all studies. Compound was dosed in a vehicle of $1 \%$ DMSO, $20 \%$ polyethylene glycol (PEG 400 ) in water, and animals were dosed by oral gavage.

Lipopolysaccharide Challenge. Rats were dosed 30 minutes prior to LPS exposure, which consisted of $0.1 \mathrm{mg} / \mathrm{ml}$ solution of Escherichia coli serotype O26:B6 LPS aerosol delivered via nebulizer at $4 \mathrm{l} / \mathrm{min}$ for 20 minutes into an anesthesia-induction chamber. Four hours later, the rats were euthanized with pentobarbital $(2.6 \mathrm{~g} / \mathrm{kg}$, i.p.) and bronchoalveolar lavage (BAL) collected by a total 21-ml wash with Dulbecco's phosphate-buffered saline, in seven 3-ml washes. Total counts and neutrophil counts were obtained as previously described (Underwood et al., 2000). Absolute neutrophils with saline exposure were typically very low and not routinely measured to reduce animal use [see Haddad et al. (2002), Nicholls et al. (2015)] for typical results; neutrophil counts in saline controls were $<1 \%$ of total). Absolute neutrophil counts following LPS exposure in vehicle-treated rats in the various studies were: $30.8 \times 10^{5} \pm 3.6$ for the dose response study; $36.1 \times 10^{5} \pm 9$ and $46 \times 10^{5} \pm 12.7$ for the fasted and fed rats, respectively, and $22.8 \times 10^{5} \pm 3.7$ in the repeat dose study.

Ozone Challenge. Animals were exposed to ozone in a purposebuilt exposure chamber for a period of 3 hours. Ozone was generated (model HTU500-G; Azco Industries, Limited, Langley, B.C., Canada) and fed into the chamber at a rate of $0.5 \mathrm{l} / \mathrm{min}$ mixed with laboratory air at $10 \mathrm{l} / \mathrm{min}$. The ozone concentration was monitored throughout the exposure period and maintained at $3 \mathrm{ppm}$ through the use of an ozone analyzer (Model 450; Advanced Instrument, Inc., Norwood, MA). At 4 hours following the completion of the ozone exposure, BAL was collected and neutrophil counts determined as described above.

Data Analysis. Data are expressed as the mean \pm standard error of the mean for total and differential cell counts in BAL fluid. Statistical analysis of cell counts was performed using a one-way analysis of variance with differences between groups evaluated employing Bonferroni's correction, with $P$ values less than 0.05 considered significant. $\mathrm{ED}_{50}$ values were determined using GraphPad Prism (v4.0 or later) by plotting neutrophil counts against $\log [$ drug conc.] and interpolating the value from half of the neutrophil count of the vehicle control group.

\section{Blood Neutrophils in the Rat}

Danirixin was given to rats ( 12 per sex per group) as a suspension at 0 (vehicle), 25, 50,150, or $500 \mathrm{mg} / \mathrm{kg}$ per day once daily for 26 weeks by oral gavage [20\% PEG and 16\% (40\% w/v, hydroxylpropyl $\beta$-cyclodextrin, $\mathrm{pH} 4.0$ ) suspension]. Six rats per sex were assigned 0 or $500 \mathrm{mg} / \mathrm{kg}$ per day
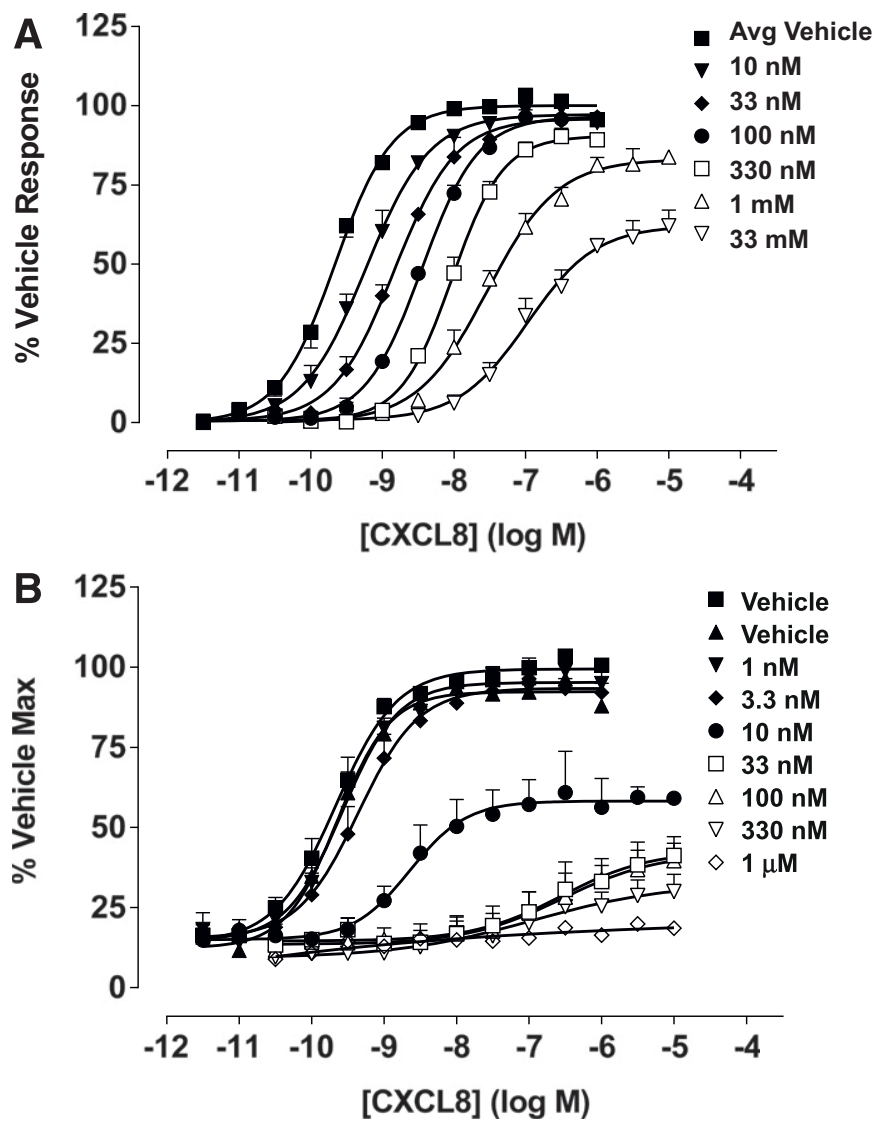

Fig. 1. Antagonism by danirixin and navirixin of CXCL8 at the CXCR2 receptor as assessed by $\mathrm{Ca}^{2+}$-flux studies in hCXCR2-expressing $\mathrm{CHO}$ cells. (A) Danirixin (B form) was preincubated for 30 minutes and shows competitive antagonism. Results are expressed as mean \pm S.E.M.; $n=3$. (B) Apparent uncompetitive antagonism by navirixin at the CXCR2 receptor under the identical experimental conditions as (A). 
to assess the reversibility of possible test item-related changes following a 6 -week recovery period. Three rats per sex were added at each dose level for toxicokinetic evaluation.

\section{Results}

\section{Potency and Kinetic Profile at Cloned Human Receptors. Binding of Danirixin in hCXCR1-CHO and hCXCR2-CHO Cell Membranes}

Binding studies were radiolabeled to assess relative affinity to expressed human CXCR1 and CXCR2. Danirixin (B form) competed with $\left[{ }^{125} \mathrm{I}\right]$ CXCL8 binding to membranes prepared from CHO stably transfected with human CXCR2 or CXCR1 cells, with $\mathrm{pIC}_{50} \mathrm{~s}$, respectively, of $7.90 \pm 0.04\left(n=3 ; \mathrm{IC}_{50} 12.5\right.$ $\mathrm{nM})$ and of $6.03 \pm 0.05(n=3)\left(\mathrm{IC}_{50} 977 \mathrm{nM}\right)$, or 78 -fold higher affinity for CXCR2. Binding affinity for CXCR2 was also assessed in the presence of $0.4 \%$ human serum albumin (hSA) to more closely approximate physiologic conditions, and danirixin competed with $\left[{ }^{125} \mathrm{I}\right]$ CXCL8 $(0.23 \mathrm{nM})$ with a $\mathrm{pIC}_{50}$ of $6.95(n=2)\left(\mathrm{IC}_{50}=113.5 \mathrm{nM}\right)$.

\section{Inhibition of CXCL-8-Induced Functional Calcium Mobilization in CHO-K1 hCXCR2 + G $\alpha 16$ Cells}

CHO-K1 cells expressing hCXCR2 $+\mathrm{G} \alpha 16$ were used to directly assess the functional potency of compounds by monitoring $\mathrm{Ca}^{2+}$ flux. Danirixin (B form) potently, competitively, and reversibly inhibited CXCL8-induced calcium mobilization. CXCL8-induced $\mathrm{Ca}^{2+}$-response curves are shown in Fig. $1 \mathrm{~A}$ in the presence of increasing danirixin concentrations. All danirixin-induced dextral displacement can be surmounted by increasing concentrations of CXCL8, achieving the maximal CXCL8 response with all but the highest concentrations of danirixin (Fig. 1A). This reduction at the high concentrations probably resulted from incomplete equilibrium at the receptor during the rapid $\mathrm{Ca}^{2+}$-flux response. Schild analysis of the concentration curve data gives a slope of 1 (data not shown), as expected for competitive inhibition, a mean $\mathrm{pA}_{2}$ of 8.18 [95\% confidence interval (CI) 8.14-8.23], or a $\mathrm{K}_{\mathrm{B}}$ of $6.5 \mathrm{nM}(n=3)$. When the assay was performed in the presence of $0.5 \% \mathrm{hSA}$, danirixin ( $\mathrm{B}$ form) had a single concentration $\mathrm{pA}_{2}$ value of 7.52 (95\% CI 7.30-7.73) or a roughly 5-fold lower potency in the presence of added protein (data not shown).

The hydrochloride salt, or A form, was evaluated separately and, as expected, had comparable functional potency, with a mean $\mathrm{pA}_{2}$ of $8.25(95 \% \mathrm{CI} 8.16-8.33)$ or a $\mathrm{K}_{\mathrm{B}}$ of $5.6 \mathrm{nM}(n=3)$. When the assay was performed in the presence of $0.5 \% \mathrm{hSA}$, calculation of potency using single-pretreatment concentrations of danirixin resulted in an apparent $\mathrm{pA}_{2}$ of $7.54(95 \% \mathrm{CI}$ 6.94-8.14; data not shown).

Additional studies were done to evaluate reversibility at the receptor by using washout studies. Danirixin B form was incubated with cells expressing hCXCR2 for a total of 90 minutes, and excess drug was washed out and cells allowed to re-equilibrate over a period of 180 minutes. Figure 2A shows that, following the washout of danirixin, CXCL8 agonist response curves overlaid the response curve obtained for the vehicle-treated cells (open symbols). These data indicate that the danirixin interaction with hCXCR2 is completely reversible within 180 minutes over the concentration range used.

Similar $\mathrm{Ca}^{2+}$-mobilization studies were carried out with navarixin to compare and contrast the kinetics and binding of the two compounds. Navarixin kinetics were assessed at both
CXCR1 (data not shown) and CXCR2 and they demonstrated reversible, competitive binding at CXCR1, as did danirixin, but somewhat different kinetics at CXCR2, consistent with previous studies suggesting noncompetitive or allosteric binding (Gonsiorek et al., 2007), as shown in Fig. 1B. Again, similar reversibility studies at CXCR2 were carried out with a 90-minute incubation and 180-minute washout period (Fig. 2B). These studies also demonstrated that navarixin could be completely removed from the receptor by washing of the cells.

\section{Selectivity: CEREP Screen and hERG Channel}

Binding studies were performed to evaluate the selectivity profile of the A form at 50 targets in the CEREP panel of receptors, ion channels, and transporters (Eurofins CEREP, Celle L'Evescault, France). GSK1325756A at a screening concentration of $1 \mu \mathrm{M}$ did not inhibit binding at any nonCXCR2 target by greater than $21 \%$.

\section{Inhibition of Agonist-Induced CD11b Expression in Whole Blood}

Engagement of the CXCR2 receptor on neutrophils by its ligand, the chemoattractant CXCL1, results in several pathways of cellular activation, including the rapid upregulation of CD11b, which, together with CD18, forms the adhesion molecule Mac-1. The ability of an antagonist to block this
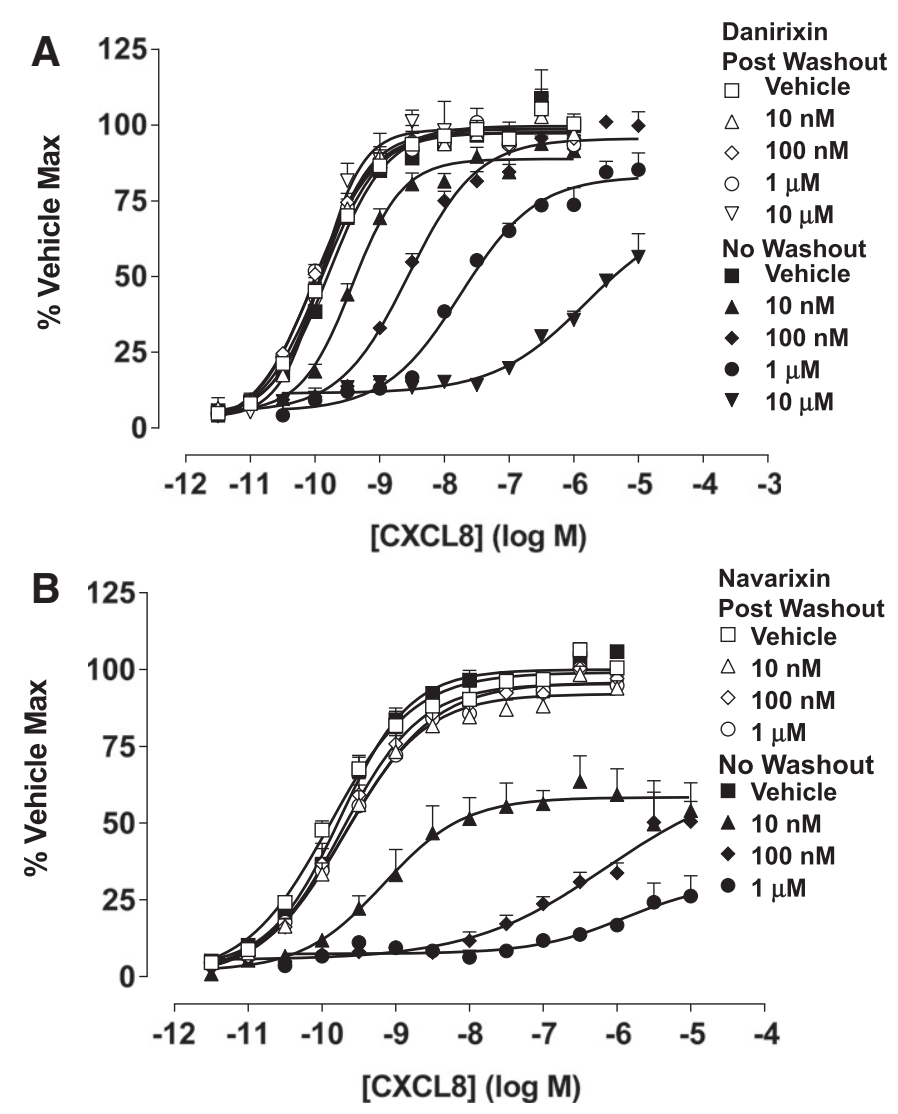

Fig. 2. Reversibility of danirixin and navirixin binding to hCXCR2-expressing $\mathrm{CHO}$ cells, as assessed by $\mathrm{Ca}^{2+}$-flux studies. (A) Danirixin (B form) binds reversibly to CXCR2. Compound was preincubated for 90 minutes, followed by a 180-minute washout before determining cellular response to CXCL8. Results are expressed as mean \pm S.E.M.; $n=3$. (B) Navirixin also binds reversibly to CXCR2 under the identical experimental conditions as in (A). 


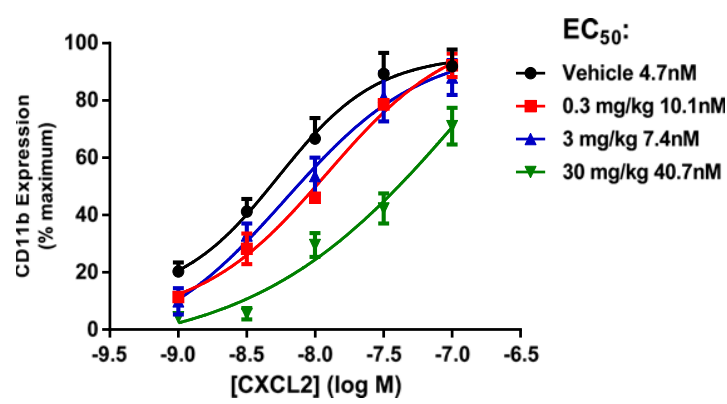

Fig. 3. CD11b expression levels on neutrophils measured by flow cytometry in rat whole blood following ex vivo challenge with CXCL2 of blood collected 1 hour after oral dosing of danirixin (B form) at $0.3,3$, and $30 \mathrm{mg} / \mathrm{kg}$ to groups of $3-5$ rats. The $\mathrm{EC}_{50}$ of CXCL2 trended to increase with increasing dose of compound.

upregulation in rat as well as human whole blood was used as a measure of functional antagonism in a physiologic setting. The identical assay can then be directly translated to a clinical setting (Nicholson et al., 2007; Lazaar et al., 2011).

Rat In Vitro Assay. Fresh rat blood was incubated with compound and then challenged with a single concentration of rat CXCL2, followed by a short incubation and assessment of surface CD11b levels by flow cytometry. GSK1325756A caused a concentration-dependent inhibition of rat neutrophil CD11b expression with a $\mathrm{pIC}_{50}=6.05, \mathrm{IC}_{50} 890 \mathrm{nM}$, and maximum inhibition of $104 \%(n=4)$ (data not shown).

Rat Ex Vivo Assay. Similar studies were carried out that used rat blood obtained following oral dosing of danirixin B form in Lewis rats. Increasing doses of danirixin caused a dose-dependent rightward shift in CXCL2 concentration-response curves compared with vehicle-treated rats, as shown in Fig. 3. The $\mathrm{EC}_{50}$ for CXCL2-induced neutrophil CD11b expression in vehicle-treated rats was $4.5 \mathrm{nM}$ and this increased to $9.4,11.9$, and $38.1 \mathrm{nM}$ at danirixin doses of $0.3,3$, and $30 \mathrm{mg} / \mathrm{kg}$, respectively. That is, the antagonist present in the blood obtained from rats dosed with compound was sufficient to reduce the effect of the agonist, making it appear less potent at the receptor.

Human In Vitro Assay. Similar studies using human whole blood and CXCL1 as the ligand are shown in Fig. 4. Danirixin shows a simple dose response with a $\mathrm{pIC}_{50}$ of $6.41 \pm 0.07, \mathrm{IC}_{50}$ $420 \mathrm{nM}$, whereas the comparator compound navirixin was more potent, with a $\mathrm{pIC}_{50}$ of $6.8, \mathrm{IC}_{50}$ of $150 \mathrm{nM}$ (data not shown).

\section{Effect on Neutrophil Influx in Rat Models}

Although there are no animal models of COPD, inhalation of LPS or ozone by humans and rats causes an inflammatory response in the lungs characterized by increases in airway neutrophils. Danirixin was therefore evaluated in several studies in two rat models of neutrophil influx, using LPS and ozone as the challenge agents.

LPS-Induced Pulmonary Neutrophilia. The ability of danirixin (B form) to block neutrophil influx to the lung following inhaled LPS challenge was evaluated in fed and fasted Lewis rats, and following multiple doses of danirixin. All studies demonstrated a dose response over the dose range used (1-30 mg/kg, by mouth). In fasted rats, the highest dose, $10 \mathrm{mg} / \mathrm{kg}$, produced a maximum $84 \%$ inhibition of neutrophils in BAL fluid, with an $\mathrm{ED}_{50}$ of $1.4 \mathrm{mg} / \mathrm{kg}$ as shown in Fig. $5 \mathrm{~A}$. In fed rats (Fig. 5B), the maximum inhibition observed was $64 \%$ at $10 \mathrm{mg} / \mathrm{kg}$ with an $\mathrm{ED}_{50}$ of $2.3 \mathrm{mg} / \mathrm{kg}$. The analysis did

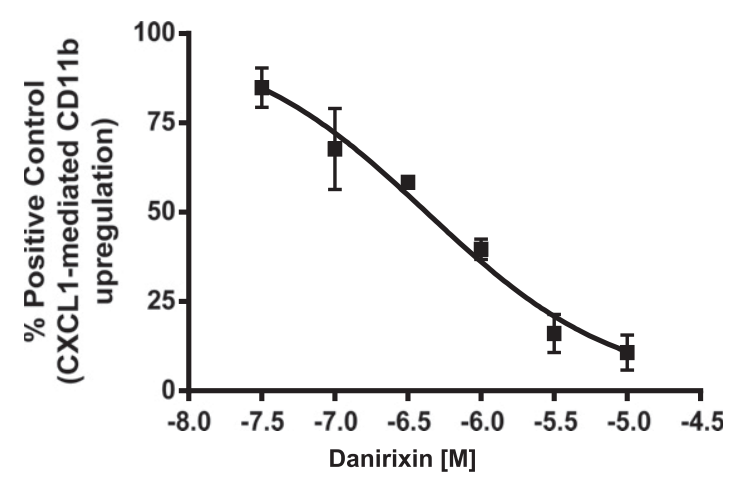

Fig. 4. Effect of danirixin (A form) on CXCL1-mediated CD11b upregulation on neutrophils in human whole blood. Data are expressed as mean (S.E.M.) using three donors.

incorporate the apparent outlier point at $3 \mathrm{mg} / \mathrm{kg}$, since it did not meet the criteria for omission. Leaving this point out results in an $\mathrm{ED}_{50}$ of $1.2 \mathrm{mg} / \mathrm{kg}$. The possibility of drug accumulation or changes in receptor function following repeated daily dosing of GSK1325756B was also assessed by dosing for 5 days, followed by a single LPS challenge. On day five, rats received the final dose 1 hour prior to LPS aerosol challenge. Rats treated for five consecutive days had a $60 \%$ inhibition of neutrophils at the single dose of $3 \mathrm{mg} / \mathrm{kg}$ recovered in BAL compared with the vehicle-treated group. A separate group of rats receiving only a single dose of danirixin 1 hour prior to LPS challenge had a 53\% inhibition of neutrophils in BAL (Fig. 6). There was no significant difference in the level of neutrophil attenuation between one and five daily doses, and thus no evidence for drug accumulation or change in receptor function.

Effect on Ozone-Induced Pulmonary Neutrophilia. A single inhaled ozone challenge over 3 hours in rats results in a similar influx of neutrophils to the lung. Danirixin (B form) dosed to fasted Lewis rats 1 hour prior to ozone exposure caused a dose-dependent inhibition of neutrophils recovered in the BAL 4 hours postchallenge. The maximum inhibition of neutrophils was $79 \%$ and the $\mathrm{ED}_{50}$ was $16.0 \mathrm{mg} / \mathrm{kg}$ (Fig. 7). Although the $\mathrm{ED}_{50}$ is considerably higher than that in the LPS model, similar shifts in potency have been observed with other anti-inflammatory mechanisms, such as p38 inhibition (Williams et al., 2008).

\section{Correlation of Rat LPS-Induced Neutrophilia and Ex-Vivo CD11b Upregulation}

The relationship between the in vivo LPS-induced neutrophilia with the ex vivo rat CXCL2-induced CD11b upregulation was evaluated. Blood levels for the in vivo LPS-induced neutrophilia were obtained after 5 hours, whereas blood levels for the samples from the ex vivo rat CXCL2-induced CD11b upregulation were obtained after 1 hour. The ex vivo $\mathrm{ED}_{50} \mathrm{~S}$ are plotted in comparison with the inhibition of neutrophils in the BAL over the same dose range as in Fig. 8, showing the same general trend over this dose range, consistent with a common mechanism for the two processes.

\section{Effects on Peripheral Hematology in Preclinical Species}

Hematological effects of danirixin in the clinic have previously been described (Miller et al., 2015). In contrast to danirixin, CXCR2 antagonists from other chemical classes 


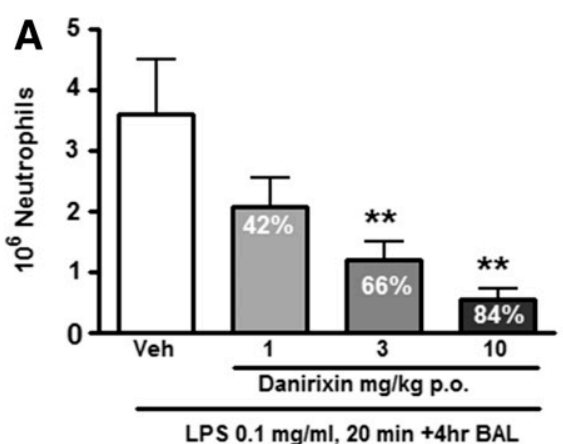

B

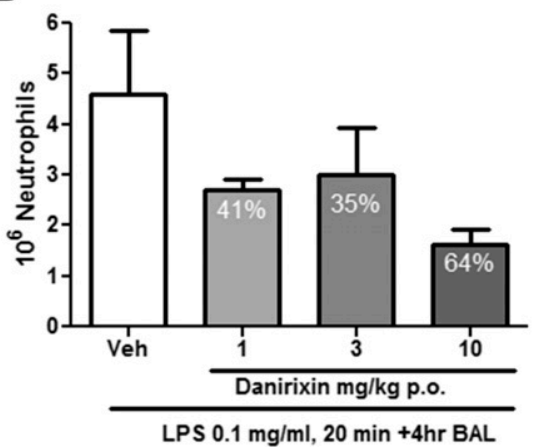

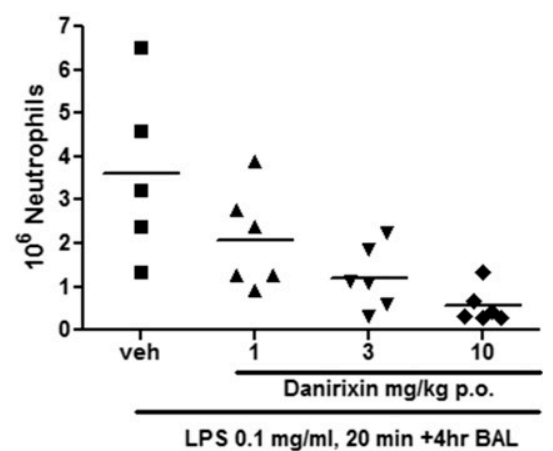

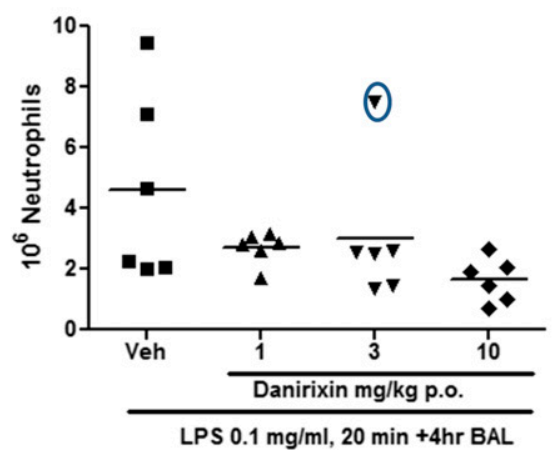

Fig. 5. Inhibition by orally administered danirixin (B form) of LPS-induced neutrophilia in bronchoalveolar lavage of (A) fasted rats or (B) fed rats Left: means and S.E.M. of groups of five rats each; right: means with individual values per rat. $* * P<0.01$. For $(\mathrm{B})$, the indicated point in on the right was removed from the analysis in the left. have demonstrated a reduction in peripheral blood neutrophils in humans in clinical studies (Kirsten et al., 2015; Rennard et al., 2015), posing a challenge to development. The preclinical hematologic effects of danirixin were also evaluated as a component of toxicology studies. In pivotal dog studies with a solution formulation, no hematologic effects were evident in dogs given $\leq 30 \mathrm{mg} / \mathrm{kg}$ per day for 7 days; however, mildly increased ( $\leq 2$-fold) mean total white blood cell and neutrophil counts were noted in male dogs given $30 \mathrm{mg} / \mathrm{kg}$ per day following 4 and 39 weeks of dosing. These changes were not considered adverse owing to lack of correlating microscopic findings and mild severity. In 7-day or 4-week studies in rats, increases in mean neutrophil and/or monocyte counts (2- to 3 -fold) occurred in females given $\geq 150 \mathrm{mg} / \mathrm{kg}$ per day and males given $1000 \mathrm{mg} / \mathrm{kg}$ per day. Effects on total white blood cell and/or neutrophil and monocytes counts were not noted in rats following 26 weeks of dosing at doses up to $500 \mathrm{mg} / \mathrm{kg}$ per day. Decreases in one or more red cell mass indices (decreased mean hemoglobin concentration, total red cell count, and hematocrit values) occurred in rats following 7 days of dosing at the highest dose $(2000 \mathrm{mg} / \mathrm{kg}$ per day) and at doses $\geq 300 \mathrm{mg} / \mathrm{kg}$ per day in dogs.

\section{Discussion}

The major findings of the studies described here are that danirixin is a potent antagonist at the CXCR2 receptor, with fully competitive and reversible kinetics at the receptor, as well as significant selectivity with respect to the CXCR1 receptor. In addition, danirixin is an effective inhibitor of
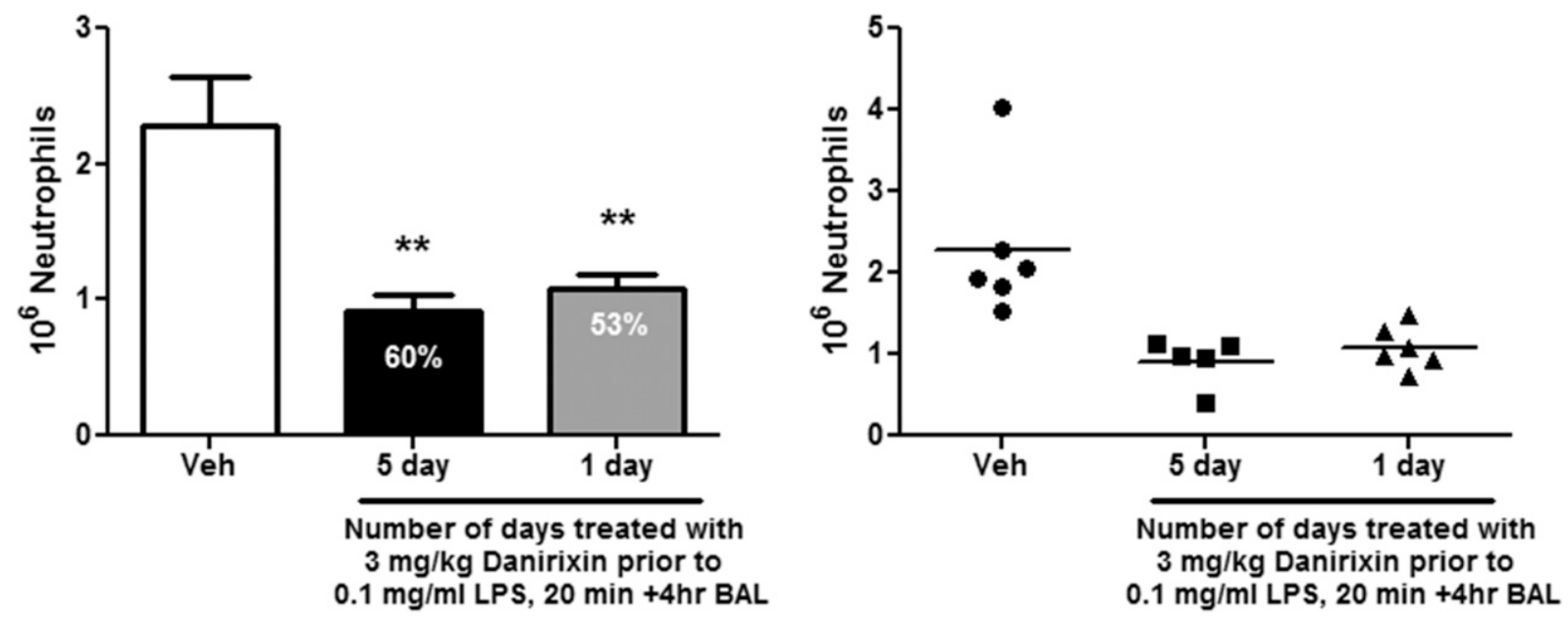

Fig. 6. Effect of repeat dosing of orally administered danirixin (B form) on inhibition of LPS-induced neutrophilia in bronchoalveolar lavage in rat. Left: means and S.E.M. of groups of five rats each; right: means with individual values per rat. 


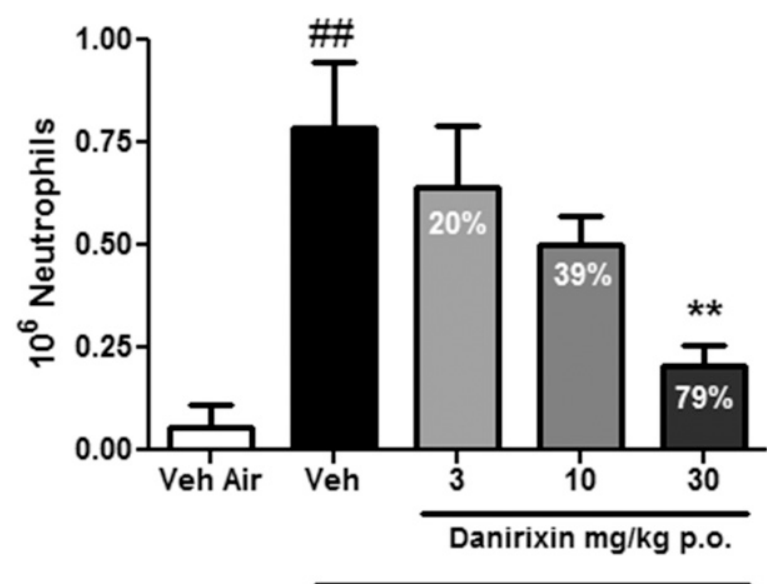

Ozone: $3 \mathrm{ppm}$, $3 \mathrm{hr}+4 \mathrm{hr}$ BAL

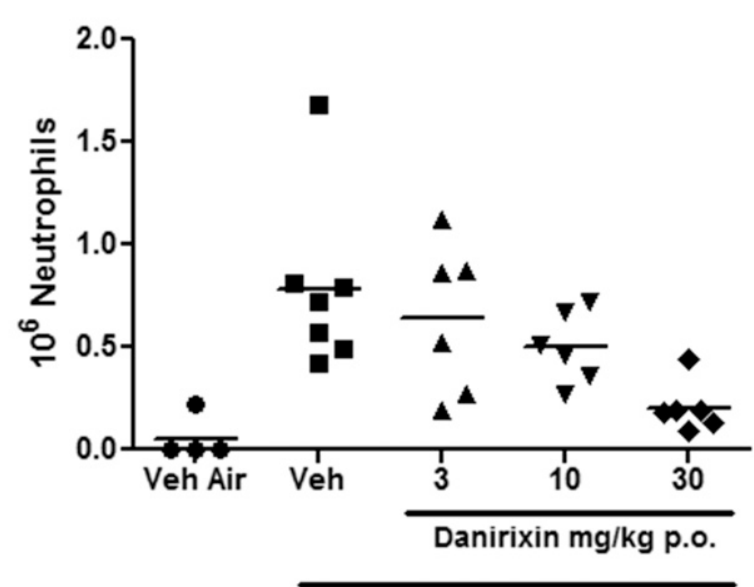

Ozone: $3 \mathrm{ppm}, 3 \mathrm{hr}+4 \mathrm{hr}$ BAL

Fig. 7. Inhibition by orally administered danirixin (B form) of ozone-induced neutrophilia in the rat. Left: means and S.E.M. from groups of five rats; right: means with individual values. ${ }^{* *} P<0.01$

pulmonary neutrophil influx following LPS or ozone challenge with no loss of activity on repeat dosing in LPS challenge.

Binding and functional assays of receptor antagonism demonstrated that danirixin is able to prevent the binding of CXCL8 to the human receptor in CHO cell membranes and to prevent $\mathrm{Ca}^{2+}$-mobilization in response to CXCL8 in intact CHO cells with comparable potency $\left(\mathrm{pIC}_{50} 7.9, \mathrm{pA}_{2} 8.2\right.$ ). Excess CXCL8 was also able to compete out the danirixin, demonstrating a binding mode that is kinetically equivalent to competitive inhibition. Danirixin was also shown to be fully reversible, completely restoring function after no more than a 180-minute washout period.

Inhibition of the CXCL1-induced CD11b upregulation has already been demonstrated to be a valuable assay for translating of efficacy to clinical studies (Nicholson et al., 2007; Lazaar et al., 2011). Using elubrixin (SB-656933), the identical whole blood assay was used in phase 1 for dose selection for an ozone challenge study (Lazaar et al., 2011). Danirixin shifts the CXCL1 response curves to the right ex vivo in rat at doses consistent with inhibition of neutrophil migration to the lung. In human blood, the $\mathrm{pIC}_{50}$ of 6.4 in the $\mathrm{CD} 11 \mathrm{~b}$ assay compared with the more potent values seen in binding or functional assays in purified systems is consistent with the moderately high protein binding observed with danirixin $(98 \%$ in human

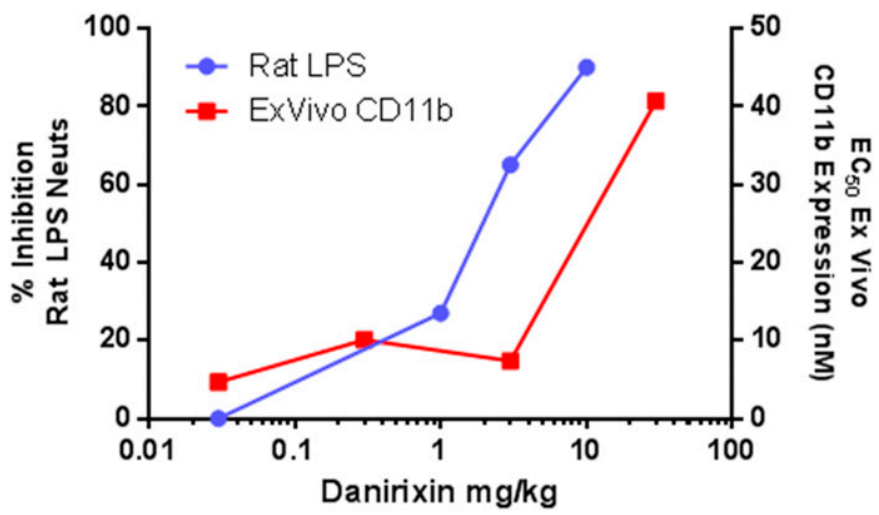

Fig. 8. Dose-related effects of danirixin on ex vivo shift in CXCL2-induced CD11b upregulation and inhibition of LPS-induced neutrophilia in the rat. plasma). This value is also consistent with the $\mathrm{pIC}_{50}$ of 6.8 $(69 \mathrm{ng} / \mathrm{ml})$ determined by pharmacokinetic/pharmacodynamic modeling in phase 1 (Miller et al., 2015).

Inhibition of pulmonary neutrophilia in challenge models by danirixin is consistent with observations of a variety of other anti-inflammatory mechanisms in these rat models, as well as inhibition of LPS challenge in human by navirixin, and of ozone challenge by the related compound elubrixin (Holz et al., 2010; Lazaar et al., 2011; Leaker et al., 2013).

The previously reported receptor characterization of other CXCR2 antagonists navarixin and AZD5069 suggest more slowly reversible kinetics compared with danirixin (Nicholls et al., 2015). This observation can potentially be rationalized in terms of slightly different binding modes. By using mutagenesis, a group from Astra-Zeneca (Nicholls et al., 2008) identified the intracellular lysine residue K320 as a key interaction for diaryl urea and AZ5069-type antagonists. These results demonstrated that different types of small-molecule antagonists bind to the same allosteric binding site located on the intracellular side of the receptor. This hypothesis was confirmed in additional studies (Salchow et al., 2010); however, these studies also revealed differences in the interactions of the lipophilic aryl moieties with the receptor. In particular, navarixin and an analog of AZ5069 showed large reductions in affinity when the alanine residue A249 was mutated to a leucine (280- and 14-fold, respectively), whereas the affinity of the danirixin analog SB-265610 was essentially unaffected. This could suggest that navarixin and AZ5069 induces a conformational change that involves A249 and this in turn is responsible for the slower off-rate of these compounds. A conformation change would also explain the apparent noncompetitive, or insurmountable, binding kinetics observed in several assays (Gonsiorek et al., 2007) for navirixin. Although navirixin is reversible, as is danirixin, the different kinetics at the receptor in functional assays may result in distinct downstream signaling that translates into differences in pharmacology.

The role of CXCR2 antagonists in altering blood neutrophil levels has been investigated since the first reports of neutropenia in human subjects treated with navirixin (Rennard et al., 2015) or AZD5069 (Kirsten et al., 2015). Neutropenia was not observed either preclinically as described here for danirixin or 
clinically with either danirixin (Miller et al., 2015) or the related compound elubrixin (Lazaar et al., 2011). The differences in the behavior of these compounds at the CXCR2 receptor, as well as selectivity with respect to CXCR1, may explain the distinct clinical observations.

It has been shown by a variety of methods that CXCR2 is required in order for release of neutrophils from the bone marrow to be maintained, and that CXCR2 and CXCR4 interact to maintain homeostasis of plasma levels (Eash et al., 2010). CXCR2 is expressed at high levels on mature neutrophils in the blood and bone marrow, and engagement of CXCR2 plays a critical role in the recruitment of neutrophils into tissues in many models of inflammation (Kobayashi, 2006). As neutrophils age, CXCR4 levels increase and CXCR2 levels decrease, leading to either a return to the bone marrow or a decrease in migration to CXCR2 ligands (Rankin, 2010). Mice engineered to have a deletion in CXCR2 showed impaired neutrophil migration (Liu et al., 2013), and hyperfunctional CXCR4 in severe combined immunodeficiency mice have accumulation of neutrophils in the bone marrow (Rankin, 2010). In the CXCR2-deficient mice, CXCL1 was not efficiently scavenged in mice with greater than $90 \%$ deletion, but in mice with a less pronounced deletion, there was no defect in scavenging (Liu et al., 2013).

A possible mechanism for the neutropenia observed would be a sufficient decrease in CXCR2 signaling to limit the neutrophils recruited from the bone marrow as CXCR4 continues to signal for return to the bone marrow. Because danirixin, navirixin, and AZD-5029 all have differing receptor potencies and kinetics, each will have differing functional blockade of CXCR2 signaling in terms of percentage of blockade, duration of blockade, and perhaps different effects on the downstream signaling.

A detailed clinical study with navirixin showed a decrease in absolute neutrophil count but no changes in bone marrow cell maturity or symptoms of reduced neutrophils (Hastrup et al., 2015), which is consistent with the hypothesis that changes in peripheral neutrophil counts are related to trafficking and not intrinsic changes in the neutrophils. Although administration of danirixin in clinical trials does not result in neutropenia or a reduction in peripheral blood neutrophils at pharmacologically effective doses up to $150 \mathrm{mg}$, reduction in peripheral blood neutrophils was observed at higher doses (Miller et al., 2015), suggesting a different but subtle effect on signaling or receptor kinetics. Additional work comparing the receptor interactions and downstream effects of the various antagonists are required to understand the molecular basis of the differences in neutropenia. Danirixin has been shown to be a selective CXCR2 antagonist that may offer benefit in diseases of excess neutrophilia, such as COPD, without concomitant neutropenia in the blood.

\section{Acknowledgments}

The authors acknowledge the contributions of Henry Sarau, Dulcie Schmidt, Michael Palovich, and Katherine Widdowson to this project

\section{Authorship Contributions}

Participated in research design: Busch-Petersen, Foley, Salmon. Conducted experiments: Foley, Hunsberger, Kilian, Burman.

Performed data analysis: Burman, Foley, Hunsberger, Kilian, Carpenter, Salmon.

Wrote or contributed to the writing of the manuscript: BuschPetersen, Foley, Mayer, Yonchuk, Carpenter, Tal-Singer.

\section{References}

Auer PL, Teumer A, Schick U, O’Shaughnessy A, Lo KS, Chami N, Carlson C, de Denus S, Dubé MP, Haessler J, et al. (2014) Rare and low-frequency coding variants in CXCR2 and other genes are associated with hematological traits. Nat Genet 46:629-634.

Aul R, Patel S, Summerhill S, Kilty I, Plumb J, and Singh D (2012) LPS challenge in healthy subjects: an investigation of neutrophil chemotaxis mechanisms involving CXCR1 and CXCR2. Int Immunopharmacol 13:225-231.

Busch-Petersen J (2006) Small molecule antagonists of the CXCR2 and CXCR1 chemokine receptors as therapeutic agents for the treatment of inflammatory diseases. Curr Top Med Chem 6:1345-1352.

Busch-Petersen J inventor and assignee (2007) Preparation of $N$-sulfonylphenyl- $N^{\prime}$ (hetero)arylureas as antagonists of chemokines, interleukin-8 (IL-8). U.S. patent 20,070,249,672A1. 2007 Oct 25.

Chapman RW, Phillips JE, Hipkin RW, Curran AK, Lundell D, and Fine JS (2009) CXCR2 antagonists for the treatment of pulmonary disease. Pharmacol Ther 121: $55-68$

Davis HM, Carpenter DC, Stahl JM, Zhang W, Hynicka WP, and Griswold DE (2000) Human granulocyte CD11b expression as a pharmacodynamic biomarker of inflammation. J Immunol Methods 240:125-132.

Dwyer MP and Yu Y (2014) CXCR2 receptor antagonists: a medicinal chemistry perspective. Curr Top Med Chem 14:1590-1605

Dwyer MP, Yu Y, Chao J, Aki C, Chao J, Biju P, Girijavallabhan V, Rindgen D, Bond R, Mayer-Ezel R, et al. (2006) Discovery of 2-hydroxy-N,N-dimethyl-3-2-[[(R)-1-(5methylfuran-2-yl)propyl]amino]-3,4-dioxocyclobut-1-enylaminobenzamide (SCH 527123): a potent, orally bioavailable CXCR2/CXCR1 receptor antagonist. J Med Chem 49:7603-7606.

Eash KJ, Greenbaum AM, Gopalan PK, and Link DC (2010) CXCR2 and CXCR4 antagonistically regulate neutrophil trafficking from murine bone marrow. $J$ Clin Invest 120:2423-2431.

Fan X, Patera AC, Pong-Kennedy A, Deno G, Gonsiorek W, Manfra DJ, Vassileva G, Zeng M, Jackson C, Sullivan L, et al. (2007) Murine CXCR1 is a functional receptor for GCP-2/CXCL6 and interleukin-8/CXCL8. J Biol Chem 282:11658-11666.

Gonsiorek W, Fan X, Hesk D, Fossetta J, Qiu H, Jakway J, Billah M, Dwyer M, Chao J, Deno G, et al. (2007) Pharmacological characterization of Sch527123, a potent allosteric CXCR1/CXCR2 antagonist. J Pharmacol Exp Ther 322:477-485.

Haddad B, McCluskie K, Birrell MA, Dabrowski D, Pecoraro M, Underwood S, Chen B, De Sanctis GT, Webber SE, Foster ML, et al. (2002) Differential effects of ebselen on neutrophil recruitment, chemokine, and inflammatory mediator expression in a rat model of lipopolysaccharide-induced pulmonary inflammation. J Immunol 169:974-982.

Haddad E-B, Salmon M, Koto H, Barnes PJ, Adcock I, and Chung KF (1996) Ozone induction of cytokine-induced neutrophil chemoattractant (CINC) and nuclear factor-k b in rat lung. inhibition by corticosteroids. FEBS Lett 379·265-268.

Hartl D, Latzin P, Hordijk P, Marcos V, Rudolph C, Woischnik M, Krauss-Etschmann S, Koller B, Reinhardt D, Roscher AA, et al. (2007) Cleavage of CXCR1 on neutrophils disables bacterial killing in cystic fibrosis lung disease. Nat Med 13:1423-1430.

Hastrup N, Khalilieh S, Dale DC, Hanson LG, Magnusson P, Tzontcheva A, Tseng J, Huyck S, Rosenberg E, and Krogsgaard K (2015) The effects of the CXCR2 antagonist, MK-7123, on bone marrow functions in healthy subjects. Cytokine $\mathbf{7 2}$ : 197-203.

Holz O, Khalilieh S, Ludwig-Sengpiel A, Watz H, Stryszak P, Soni P, Tsai M, Sadeh J, and Magnussen H (2010) SCH527123, a novel CXCR2 antagonist, inhibits ozoneinduced neutrophilia in healthy subjects. Eur Respir $J$ 35:564-570.

Kirsten AM, Förster K, Radeczky E, Linnhoff A, Balint B, Watz H, Wray H, Salkeld L, Cullberg M, and Larsson B (2015) The safety and tolerability of oral AZD5069, a selective CXCR2 antagonist, in patients with moderate-to-severe COPD. Pulm Pharmacol Ther 31:36-41.

Kobayashi Y (2006) Neutrophil infiltration and chemokines. Crit Rev Immunol 26 307-316.

Lazaar AL, Sweeney LE, MacDonald AJ, Alexis NE, Chen C, and Tal-Singer R (2011) SB-656933, a novel CXCR2 selective antagonist, inhibits ex vivo neutrophil activation and ozone-induced airway inflammation in humans. $\mathrm{Br}$ J Clin Pharmacol 72:282-293.

Leaker BR, Barnes PJ, and O'Connor B (2013) Inhibition of LPS-induced airway neutrophilic inflammation in healthy volunteers with an oral CXCR2 antagonist. Respir Res 14:137-146.

Liu L, Li M, Spangler LC, Spear C, Veenstra M, Darnall L, Chang C, Cotleur AC, and Ransohoff RM (2013) Functional defect of peripheral neutrophils in mice with induced deletion of CXCR2. Genesis 51:587-595.

Miller BE, Mistry S, Smart K, Connolly P, Carpenter DC, Cooray H, Bloomer JC, Tal-Singer R, and Lazaar AL (2015) The pharmacokinetics and pharmacodynamics of danirixin (GSK1325756)-a selective CXCR2 antagonist-in healthy adult subjects. BMC Pharmacol Toxicol 16:18-30.

Nagarkar DR, Wang Q, Shim J, Zhao Y, Tsai WC, Lukacs NW, Sajjan U, and Hershenson MB (2009) CXCR2 is required for neutrophilic airway inflammation and hyperresponsiveness in a mouse model of human rhinovirus infection. J Immunol $\mathbf{1 8 3}$ $6698-6707$

Nicholls DJ, Tomkinson NP, Wiley KE, Brammall A, Bowers L, Grahames C, Gaw A Meghani P, Shelton P, Wright TJ, et al. (2008) Identification of a putative intracellular allosteric antagonist binding-site in the CXC chemokine receptors 1 and 2. Mol Pharmacol 74:1193-1202.

Nicholls DJ, Wiley K, Dainty I, MacIntosh F, Phillips C, Gaw A, and Mårdh CK (2015) Pharmacologic characterization of AZD5069, a slowly reversible CXC receptor 2 antagonist. J Pharmacol Exp Ther 353:340-350.

Nicholson GC, Tennant RC, Carpenter DC, Sarau HM, Kon OM, Barnes PJ, Salmon M, Vessey RS, Tal-Singer R, and Hansel TT (2007) A novel flow cytometric assay of human whole blood neutrophil and monocyte CD11b levels: upregulation by chemokines is related to receptor expression, comparison with neutrophil shape 
change, and effects of a chemokine receptor (CXCR2) antagonist. Pulm Pharmacol Ther 20:52-59.

Podolin PL, Bolognese BJ, Foley JJ, Schmidt DB, Buckley PT, Widdowson KL, Jin Q, White JR, Lee JM, Goodman RB, et al. (2002) A potent and selective nonpeptide antagonist of CXCR2 inhibits acute and chronic models of arthritis in the rabbit. $J$ Immunol 169:6435-6444.

Qiu Y, Zhu J, Bandi V, Atmar RL, Hattotuwa K, Guntupalli KK, and Jeffery PK (2003) Biopsy neutrophilia, neutrophil chemokine and receptor gene expression in severe exacerbations of chronic obstructive pulmonary disease. Am J Respir Crit Care Med 168:968-975.

Quint JK and Wedzicha JA (2007) The neutrophil in chronic obstructive pulmonary disease. J Allergy Clin Immunol 119:1065-1071.

Rankin SM (2010) The bone marrow: a site of neutrophil clearance. J Leukoc Biol 88 241-251

Raghuwanshi SK, Su Y, Singh V, Haynes K, Richmond A, and Richardson RM (2012) The chemokine receptors CXCR1 and CXCR2 couple to distinct G protein-coupled receptor kinases to mediate and regulate leukocyte functions. J Immunol 189:2824-2832.

Rennard SI, Dale DC, Donohue JF, Kanniess F, Magnussen H, Sutherland ER, Watz H, Lu S, Stryszak P, Rosenberg E, et al. (2015) CXCR2 antagonist MK-7123. A phase 2 proof-of-concept trial for chronic obstructive pulmonary disease. Am J Respir Crit Care Med 191:1001-1011.

Salchow K, Bond ME, Evans SC, Press NJ, Charlton SJ, Hunt PA, and Bradley ME (2010) A common intracellular allosteric binding site for antagonists of the CXCR2 receptor. Br J Pharmacol 159:1429-1439.

Sarau HM, Griswold DE, Potts W, Foley JJ, Schmidt DB, Webb EF, Martin LD, Brawner ME, Elshourbagy NA, Medhurst AD, et al. (1997) Nonpeptide tachykinin receptor antagonists: I. Pharmacological and pharmacokinetic characterization of SB 223412, a novel, potent and selective neurokinin-3 receptor antagonist. $J$ Pharmacol Exp Ther 281:1303-1311.

Stadtmann A and Zarbock A (2012) CXCR2: From bench to bedside. Front Immunol 3:263.

Underwood DC, Osborn RR, Bochnowicz S, Webb EF, Rieman DJ, Lee JC, Romanic AM, Adams JL, Hay DW, and Griswold DE (2000) SB 239063, a p38 MAPK inhibitor, reduces neutrophilia, inflammatory cytokines, MMP-9, and fibrosis in lung. Am J Physiol Lung Cell Mol Physiol 279:L895-L902.

Thatcher TH, McHugh NA, Egan RW, Chapman RW, Hey JA, Turner CK, Redonnet MR, Seweryniak KE, Sime PJ, and Phipps RP (2005) Role of CXCR2 in cigarette smoke-induced lung inflammation. Am J Physiol Lung Cell Mol Physiol 289 L322-L328.

Wareing MD, Shea AL, Inglis CA, Dias PB, and Sarawar SR (2007) CXCR2 is required for neutrophil recruitment to the lung during influenza virus infection, but is not essential for viral clearance. Viral Immunol 20:369-378.

Williams AS, Issa R, Durham A, Leung SY, Kapoun A, Medicherla S, Higgins LS, Adcock IM, and Chung KF (2008) Role of p38 mitogen-activated kinase in ozoneinduce airway hyperresponsiveness and inflammation. Eur $J$ Pharmacol 609: 155-156. Erratum in Eur J Pharmacol (2009) 609:155-156.

Address correspondence to: Dr. Ruth Tal-Singer, Respiratory Therapy Area Unit, GSK R\&D, 709 Swedeland Road, King of Prussia, PA 19406. E-mail: Ruth.M.Tal-Singer@gsk.com. 